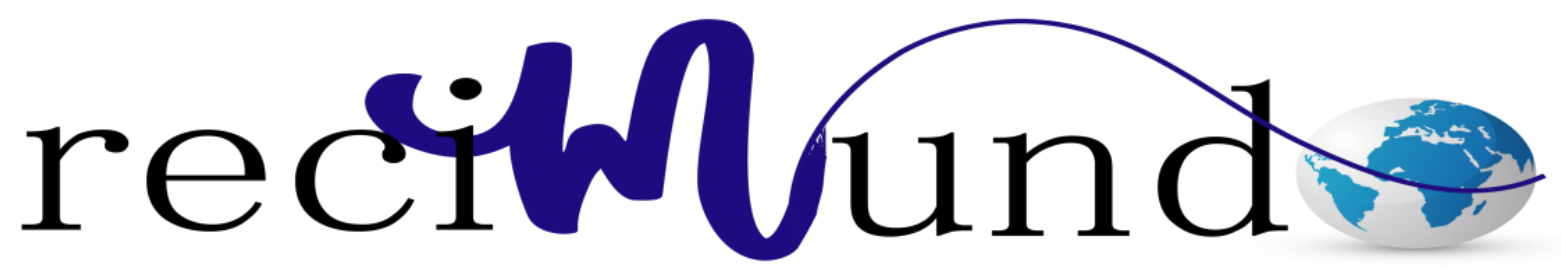

Revista Científica Mundo de la Investigación y el Conocimiento

Colombo Efraín Arteaga Delgado a ; Raúl Ivan Casahualpa Leones ${ }^{\text {b }}$; Fabrico Wladimir Gutiérrez Rivera ${ }^{c}$; Jenniffer Betzabe Santistevan Holguin ${ }^{\text {d; }}$ Yuly Simone Mitte Baque ${ }^{e}$; Alexandra Anabell Figueroa Parrales ${ }^{f}$

Enfoque sobre los factores de riesgo más relevantes de la diabetes en niños Focus on the most relevant risk factors of diabetes in children

Revista Científica Mundo de la Investigación y el Conocimiento. Vol. 2 núm.4, Octubre, ISSN: 2588-073X, 2018, pp. 270-297

DOI: 10.26820/recimundo/2.(4).octubre.2018.270-297

URL: http://www.recimundo.com/index.php/es/article/view/353

Editorial Saberes del Conocimiento

Recibido: $15 / 07 / 2018 \quad$ Aceptado: 05/08/2018 Publicado: 30/10/2018

Correspondencia: editor@ recimundo.com

a. Médico Residente de Traumatología Hospital Dr Gustavo Domínguez Zambrano

b. Hospital Dr gustavo Domínguez Zambrano; Residente de traumatología

c. Medico Asistencial en funciones Hospitalarias; Hospital Básico Jipijapa

d. Medico Asistencial en funciones Hospitalarias; Hospital Básico Jipijapa

e. Médico Residente Hospital Dr Gustavo Dominguez

f. Médico General en funciones hospitalarias; Centro Clínico Quirugico Ambulatorio Hospital del Día Santo Domingo 


\section{Enfoque sobre los factores de riesgo más relevantes de la diabetes en niños}

Vol. 2, núm. 4., (2018)

Colombo Efraín Arteaga Delgado; Raúl Ivan Casahualpa Leones; Fabrico Wladimir Gutiérrez Rivera; Jenniffer Betzabe Santistevan Holguin; Yuly Simone Mitte Baque; Alexandra Anabell

Figueroa Parrales

\section{RESUMEN}

En los niños la Diabetes Mellitus del tipo 1 se presenta por los cambios perjudiciales en los estilos de vida los mismos que están estableciendo un perfil epidemiológico permitiendo la presencia de distintos factores de riesgo, caracterizado por el sedentarismo en la actualidad una de las principales fuentes de amenaza para la salud de los niños, los malos hábitos alimentarios nutricionales e inadecuados, la obesidad en la población pediátrica por los antecedentes familiares de obesidad, el sobrepeso, los problemas cardiovasculares, la hipertensión. La diabetes en niños se ha identificado como un problema global que se ha complicado por varios factores ambientales y genéticos, las enfermedades crónicas no transmisibles (ECNTs). Estas patologías, que representan una importante carga en morbilidad y mortalidad en la edad adulta, se originan en gran parte, muy precozmente en la etapa infantil, incluso antes, en la etapa fetal, y se incrementan durante toda la vida. Por lo que es de vital importancia la identificación de los factores de riesgo, debe formar parte de las estrategias de prevención para asegurar que la población infantil llegue a ser adulta sin enfermedades crónicas y con adecuada calidad de vida.

Palabras claves: Diabetes Mellitus del tipo 1, sedentarismo, malos hábitos alimentarios, la obesidad, problemas cardiovasculares. 


\section{Enfoque sobre los factores de riesgo más relevantes de la diabetes en niños}

Vol. 2, núm. 4., (2018)

Colombo Efraín Arteaga Delgado; Raúl Ivan Casahualpa Leones; Fabrico Wladimir Gutiérrez Rivera; Jenniffer Betzabe Santistevan Holguin; Yuly Simone Mitte Baque; Alexandra Anabell Figueroa Parrales

\section{ABSTRACT}

In children Diabetes Mellitus type 1 is presented by the deleterious changes in lifestyles that are establishing an epidemiological profile allowing the presence of different risk factors, characterized by a sedentary lifestyle at present one of the main sources of threat to the health of children, poor nutritional and inadequate eating habits, obesity in the pediatric population due to family history of obesity, overweight, cardiovascular problems, hypertension. Diabetes in children has been identified as a global problem that has been complicated by several environmental and genetic factors, chronic noncommunicable diseases (NCDs). These pathologies, which represent an important burden in morbidity and mortality in adulthood, originate in large part, very early in childhood, even before, in the fetal stage, and increase throughout life. So it is vitally important to identify risk factors, should be part of prevention strategies to ensure that the child population becomes adult without chronic diseases and with adequate quality of life.

Keywords: Diabetes Mellitus type 1, sedentary lifestyle, poor eating habits, obesity, cardiovascular problems. 


\section{Enfoque sobre los factores de riesgo más relevantes de la diabetes en niños}

Vol. 2, núm. 4., (2018)

Colombo Efraín Arteaga Delgado; Raúl Ivan Casahualpa Leones; Fabrico Wladimir Gutiérrez Rivera; Jenniffer Betzabe Santistevan Holguin; Yuly Simone Mitte Baque; Alexandra Anabell

Figueroa Parrales

\section{Introducción.}

Hasta hace unos cuantos años, la mayoría de los niños se diagnosticaban con diabetes tipo 1 ocasionada por deficiencia total en la secreción de insulina, en tanto que la diabetes mellitus tipo 2 prevalecía en adultos y ancianos. En los últimos 10 a 20 años se ha observado un alarmante incremento en la prevalencia de diabetes mellitus tipo 2 en los centros de diabetes pediátricos de todo el mundo. Blommgarden, (2004) y Botero, Wolfsdorf, (2005).

Existen dos tipos principales de diabetes mellitus: la primaria y la secundaria. Dentro de la primaria están la diabetes tipo I la cual se manifiesta en la niñez y los primeros años de la adolescencia y donde el páncreas del paciente no produce insulina en lo absoluto. Estos pacientes son insulino dependientes por lo que deberá inyectarse insulina una o más veces al día para vivir, Gagliardino, (1997).

Entre los tipos de diabetes se destacan, la diabetes mellitus tipo 1 (DM-1), la tipo 2 (DM2) y la diabetes mellitus gestacional (DMG). La primera se desarrolla en niños y adolescentes, tiende a ser dependiente de la insulina y propensa a complicaciones como la cetoacidosis; la segunda es más frecuente en personas obesas que presentan resistencia a la insulina (RI), puede tratarse con hipoglucemiantes orales y no es propensa a la cetoacidosis; comprende del 90-95\% de todos los casos de diabetes. Es más frecuente en personas de edad media que sobrepasan los 40 años, Almaguer, et al. (2012).

La Diabetes Mellitus tipo $1(\mathrm{DM} 1)$ es una de las patologías más estudiadas en la actualidad, no solo por el aumento de su incidencia, sino también por su aparición a edades cada 


\section{Enfoque sobre los factores de riesgo más relevantes de la diabetes en niños}

Vol. 2, núm. 4., (2018)

Colombo Efraín Arteaga Delgado; Raúl Ivan Casahualpa Leones; Fabrico Wladimir Gutiérrez Rivera; Jenniffer Betzabe Santistevan Holguin; Yuly Simone Mitte Baque; Alexandra Anabell Figueroa Parrales

vez más tempranas. La DM1 es una de las enfermedades autoinmunes más estudiadas en la actualidad, no solo por su complejidad genética sino por su susceptibilidad a factores ambientales. Semejante a su contraparte Tipo 2, la DM1 se caracteriza por un defecto en el metabolismo de los carbohidratos, pero debido a la destrucción de las células $\beta$ del páncreas mediado por mecanismos autoinmunes, manifestándose como ausencia absoluta de insulina, Aguirre, et al. (2012).

La diabetes en niños se ha identificado como un problema global que se ha complicado por varios factores ambientales y genéticos. Las estimaciones recientes sugieren que al menos la mitad de la población pediátrica con diabetes pertenece al tipo 2. McKnight, et al. (2005) y Ludwig, Ebbeling, (2001).

La Diabetes Mellitus (DM) es una enfermedad sistémica que describe un desorden metabólico de múltiples etiologías, caracterizado por hiperglucemia crónica con disturbios en el metabolismo de los carbohidratos, grasas y proteínas; resulta de defectos en la secreción y/o en la acción de la insulina, Padrón, (2008) y OPS, (2008).

En la publicación de la $5^{\mathrm{a}}$ edición del Atlas de Diabetes se establece estimaciones sobre los datos actuales de DM y las previsiones para el año 2030. Las nuevas cifras estiman que en el año 2011, había 366 millones de personas con DM en el mundo y que esta cifra se incrementará hasta 552 millones en el año 2030 siendo los países de bajos y medios ingresos los que se enfrentan al mayor crecimiento de la enfermedad, IDF. Atlas Diabetes (2011). 


\section{Enfoque sobre los factores de riesgo más relevantes de la diabetes en niños}

Vol. 2, núm. 4., (2018)

Colombo Efraín Arteaga Delgado; Raúl Ivan Casahualpa Leones; Fabrico Wladimir Gutiérrez Rivera; Jenniffer Betzabe Santistevan Holguin; Yuly Simone Mitte Baque; Alexandra Anabell

Figueroa Parrales

En el mundo hay 490.100 menores de 14 años con DM1 y cada año se diagnostican 77.800 casos en esta franja de edad. La DM1 es una de las enfermedades crónicas más comunes en la infancia y el número de niños que la desarrollan está aumentando, especialmente entre los niños más pequeños y en algunas regiones de Europa central y oriental. El aumento total anual de la tasa de DM1 se estima en un 3\%. También aumenta la incidencia y prevalencia de DM2 en edad infantil y en la adolescencia, IDF. Atlas Diabetes (2011).

La DMG se define como cualquier grado de intolerancia a la glucosa con inicio o reconocida por primera vez durante el embarazo. Aunque la mayoría de los casos se resuelven con el parto, la definición se aplica si persiste o no después de la gestación. Afecta alrededor del 7\% de todos los embarazos, ADA, (2008).

La obesidad es una enfermedad crónica, compleja y multifactorial que suele iniciarse en la infancia y la adolescencia. Desde 1998 la OMS considera la obesidad una epidemia global. En la actualidad es un creciente e importante problema de salud pública al ser un factor común de riesgo para patologías como la diabetes, la enfermedad cardiovascular y la hipertensión arterial (HTA), Fernández, (2005).

Actualmente muchas enfermedades relacionadas con la obesidad consideradas frecuentemente en adultos se están reportando en niños con una tendencia cada vez mayor. Por ejemplo, la diabetes tipo 2, antes llamada diabetes del adulto, ahora aparece en niños, alrededor de los doce años. Lo que indica que los niños tienen que hacer a esta enfermedad por un período más extenso, sin contar la implicancia económica. Se ha reportado que los gastos en atenciones médicas y en medicinas en adultos obesos fue de 36 y $77 \%$ más altos que en las personas no 


\section{Enfoque sobre los factores de riesgo más relevantes de la diabetes en niños}

Vol. 2, núm. 4., (2018)

Colombo Efraín Arteaga Delgado; Raúl Ivan Casahualpa Leones; Fabrico Wladimir Gutiérrez Rivera; Jenniffer Betzabe Santistevan Holguin; Yuly Simone Mitte Baque; Alexandra Anabell Figueroa Parrales

obesas, respectivamente. Wang et al. señalan que los gastos hospitalarios para el tratamiento de las enfermedades vinculadas con la obesidad infantil se incrementaron de \$35 millones a \$127 millones de 1979-1981 a 1997-1999. Por lo tanto, la alta prevalencia de obesidad infantil y sus consecuencias obligan a prestar extrema atención a este problema de Salud Pública, Del Águila, C., (2017).

\section{Metodología.}

Para la ejecución del presente trabajo científico se efectúo un análisis de la literatura más próxima al enfoque sobre los factores de riesgo más relevantes de la diabetes en niños. En esta investigación, se consideró, información confiable respecto a trabajos plasmados, tratados referente a este tema, se utilizó información bibliográfica, consultas electrónicas, misma que se la obtuvo de libros electrónicos, artículos científicos y revistas.

\section{Desarrollo.}

Enfoque sobre los factores de riesgo

Entre los factores de riesgo están: la obesidad, la edad mayor de 25 años, historia previa de anomalías del metabolismo de la glucosa o de problemas obstétricos, familiares de primer grado con diabetes o miembros de grupos étnicos con alta prevalencia de la enfermedad. La diabetes durante el embarazo también provoca trastornos obstétricos como macrosomía y prematuridad que incrementan el riesgo de hiperbilirrubinemia, hipocalcemia y distrés respiratorio en los niños, Chukwueke y Cordero, (2010). 


\section{Enfoque sobre los factores de riesgo más relevantes de la diabetes en niños}

Vol. 2, núm. 4., (2018)

Colombo Efraín Arteaga Delgado; Raúl Ivan Casahualpa Leones; Fabrico Wladimir Gutiérrez Rivera; Jenniffer Betzabe Santistevan Holguin; Yuly Simone Mitte Baque; Alexandra Anabell

Figueroa Parrales

El World Health Report 2002, mostró con detalle cómo unos pocos e importantes factores de riesgo era responsables de una proporción significativa de todas las muertes y patologías en la mayor parte de los países estudiados. En efecto, seis de los siete más destacados factores de riesgo para una muerte prematura están correlacionados con la dieta y la actividad física (tensión arterial, colesterol, índice de masa corporal, ingestión insuficiente de fruta y de verdura, inactividad física, consumo excesivo de alcohol), WHO, (2002).

Los determinantes fundamentales de estos factores de riesgo son en su mayor parte los mismos: cambios producidos en la dieta conducentes a una mayor densidad energética y donde la grasa y el azúcar añadido tienen un papel importante, incremento de la ingestión de grasa saturada (sobre todo a partir de alimentos de origen animal) y de grasa hidrogenada, reducción del consumo de carbohidratos complejos y fibra así como una reducción en paralelo de la ingestión de frutas y verduras. Todo ello con el aumento de los tamaños de las porciones de los alimentos, sobre todo en niños y jóvenes en los que la inactividad física juega un papel predominante, Martínez, et al. (2013).

El incremento de la prevalencia de sobrepeso en niños, estimado actualmente en $25 \%$, tiene una función decisiva en el reciente aumento de diabetes mellitus tipo 2 en la población pediátrica. Además de la obesidad, otros factores de riesgo importantes incluyen: origen étnico, edad (edad promedio al momento del diagnóstico entre 12 y 16 años, y la edad más temprana de diagnóstico en una niña india Pima), sexo (más frecuente en mujeres), modo de vida sedentario, antecedentes familiares y perinatales, Ludwig, Ebbeling, (2001) y ADA. (2000). 


\section{Enfoque sobre los factores de riesgo más relevantes de la diabetes en niños}

Vol. 2, núm. 4., (2018)

Colombo Efraín Arteaga Delgado; Raúl Ivan Casahualpa Leones; Fabrico Wladimir Gutiérrez Rivera; Jenniffer Betzabe Santistevan Holguin; Yuly Simone Mitte Baque; Alexandra Anabell Figueroa Parrales

En un estudio de niños obesos el LDL fue significativamente más alto y los niveles de HDL menores con relación a los no obesos, $19 \%$ de los niños con circunferencia abdominal > al PC 90 tenían 2 o más factores de riesgo comparado con el 9\% de niños en $\mathrm{Pc} \leq$ al 90 . Concluyen que la circunferencia abdominal ajustada según edad y sexo, se asocia a factores de riesgo cardiovascular, resulta útil en la práctica clínica y su uso es apropiado para la evaluación de niños con insulinoresistencia u otras manifestaciones asociadas al SM, Maffeis, et al. (2001) y Morrison, (2000).

Las alteraciones de la salud en los niños con obesidad y sobrepeso son mayores cifras de presión arterial sistólica, dislipidemia y glucosa, comparados con los niños de peso normal, Chu, (2001).

Es así como la mayor prevalencia de síndrome metabólico y, por lo tanto, los factores de riesgo cardiovasculares se presentan a edades más tempranas. Taksali et al. (2004).

El exceso de peso es un proceso gradual que suele iniciarse en la infancia y la adolescencia a partir de un desequilibrio entre la ingestión y gasto de energía, sumado a una predisposición genética expresada en una serie polimorfismos. Además, diversos cambios epigenéticos ocurren especialmente durante el embarazo, la infancia y la pubertad. Dichos cambios determinan una "memoria" de las experiencias tempranas, desencadenantes reversibles y susceptibilidad a la enfermedad en la vida adulta. De hecho, pueden ser heredadas a través de las divisiones de las células somáticas y potencialmente adquiridas a través de varias generaciones (epimutaciones), Manco, Dallapiccola, (2012). 


\section{Enfoque sobre los factores de riesgo más relevantes de la diabetes en niños}

Vol. 2, núm. 4., (2018)

Colombo Efraín Arteaga Delgado; Raúl Ivan Casahualpa Leones; Fabrico Wladimir Gutiérrez Rivera; Jenniffer Betzabe Santistevan Holguin; Yuly Simone Mitte Baque; Alexandra Anabell

Figueroa Parrales

Como factor de riesgo prevalente en niños y adolescentes. La hipertensión acelera el desarrollo de la enfermedad coronaria y contribuye significativamente a la patogénesis de accidentes cerebrovasculares, insuficiencia cardíaca e insuficiencia renal. La elevación de la presión arterial en edades tempranas, es predictora de valores superiores en etapas más tardías de la vida; y debido a que es usualmente asintomática, los efectores de salud tienen gran responsabilidad en la identificación de individuos con riesgo, Williams, et al. (2002).

Los estudios han demostrado la estrecha relación entre obesidad, diabetes mellitus tipo 2, hipertensión arterial, litiasis vesicular y osteoartrosis, que a su vez ocasionan mayor gasto en los sistemas de salud, incrementan los días laborales perdidos, reducen la calidad de vida e incrementan la mortalidad en la población. Estos resultados reportados a nivel mundial crean la necesidad de enfocar la atención en la prevención primaria, proponer estrategias para cambiar los estilos de alimentación e incrementar la actividad física en los niños y adolescentes. Luna, et al. (2007).

La obesidad en escolares es un problema mundial que implica dislipidemia, resistencia periférica a la acción de la insulina y de leptina, hipertensión arterial y diabetes, lo que genera mayor riesgo cardiovascular, Godínez, et al. (2002) y Hernández, et al. (2003).

La alta prevalencia de complicaciones metabólicas en niños y adolescentes obesos, condicionadas fundamentalmente por el grado de obesidad, alguna de las cuales ya estaban presentes en estadios leves. El 22\% de los niños obesos severos, presentaba valores alterados de colesterol; $18 \%$ de LDL; $44 \%$ de trigliceridos, $75 \%$ de HDL; $21 \%$ de TAs y $13 \%$ de TAd, fue demostrado por Barja, et al. (2004). 


\section{Enfoque sobre los factores de riesgo más relevantes de la diabetes en niños}

Vol. 2, núm. 4., (2018)

Colombo Efraín Arteaga Delgado; Raúl Ivan Casahualpa Leones; Fabrico Wladimir Gutiérrez Rivera; Jenniffer Betzabe Santistevan Holguin; Yuly Simone Mitte Baque; Alexandra Anabell Figueroa Parrales

No sólo disminuye la calidad de vida sino que afecta la productividad y aumenta los costos de atención médica a la población. La diabetes y las enfermedades cardiovasculares se han constituido en las principales causas de morbilidad y mortalidad de la población general en un gran número de países, Calzada, (2003).

La obesidad durante la infancia está influenciada por factores genéticos, epigenéticos, conductuales y ambientales. Entre éstos, los factores conductuales y ambientales son más fácilmente modificables durante la infancia, por lo que estos son el foco de las intervenciones clínicas, de ahí la importancia de identificar factores de riesgo de obesidad en la población pediátrica. Estos factores de riesgo son los antecedentes familiares de obesidad, los malos hábitos de alimentación y el sedentarismo, entre otros, Del Águila, C., (2017).

Los efectos adversos y los riesgos de la obesidad para la salud en etapas tempranas de la vida incluyen a corto plazo tanto problemas físicos como psicosociales. Estudios longitudinales sugieren que la obesidad infantil, después de los 3 años de edad, se asocia a largo plazo con un mayor riesgo de obesidad en la edad adulta y con un aumento en la morbilidad y mortalidad; persistencia de los trastornos metabólicos asociados, un aumento del riesgo cardiovascular y de algunos tipos de cáncer, Aranceta, et al. (2005).

En los últimos años se notó un aumento en la incidencia de diabetes tipo II en adolescentes de EEUU, asociado al aumento de la prevalencia de la obesidad severa. La obesidad juega un rol fundamental con relación al síndrome de insulinoresistencia. La resistencia del cuerpo a las acciones de la insulina, resulta en una producción aumentada de esta hormona por el páncreas, llevando a un estado de hiperinsulinemia. La obesidad presente en los niños, 


\section{Enfoque sobre los factores de riesgo más relevantes de la diabetes en niños}

Vol. 2, núm. 4., (2018)

Colombo Efraín Arteaga Delgado; Raúl Ivan Casahualpa Leones; Fabrico Wladimir Gutiérrez Rivera; Jenniffer Betzabe Santistevan Holguin; Yuly Simone Mitte Baque; Alexandra Anabell

Figueroa Parrales

frecuentemente precede a este estado. La American Heart Association aconseja que pese a que las alteraciones en los niveles de glucosa pueden no estar presentes, el hecho de manifestar otros factores relacionados al SM tales como, obesidad, dislipidemias, hipertensión arterial, etc., pueden implicar el riesgo futuro de desarrollar enfermedad cardiovascular y diabetes, Steinberger, Stephen, (2003).

Un tema interesante es analizar el periodo de adiposidad de rebote. En general la masa corporal aumenta de manera rápida durante el primer año de vida y posteriormente disminuye hasta los seis años y después aumenta de nuevo. Los niños que desarrollan obesidad tiene un incremento prematuro de esa curva, el cual es llamado periodo de rebote de adiposidad, esto permite plantear estrategias de prevención para este grupo etario Rolland, et al. (2006).

Dentro de los factores que condicionan este rebote de adiposidad, se considera, según diversos estudios, el exceso de proteínas al principio de la vida. Se ha observado que los niños que consumían más proteína tenían una rápida velocidad de crecimiento y por lo tanto mayor riesgo a obesidad, Michaelsen, Greer, (2014) y Hornell, et al. (2013).

La obesidad es una enfermedad crónica, compleja y multifactorial que suele iniciarse en la infancia y la adolescencia. Desde 1998 la OMS considera la obesidad una epidemia global. En la actualidad es un creciente e importante problema de salud pública al ser un factor común de riesgo para patologías como la diabetes, la enfermedad cardiovascular y la hipertensión arterial (HTA), WHO, (1998), Serra, et al. (2003), Dietz, (1998). 


\section{Enfoque sobre los factores de riesgo más relevantes de la diabetes en niños}

Vol. 2, núm. 4., (2018)

Colombo Efraín Arteaga Delgado; Raúl Ivan Casahualpa Leones; Fabrico Wladimir Gutiérrez Rivera; Jenniffer Betzabe Santistevan Holguin; Yuly Simone Mitte Baque; Alexandra Anabell Figueroa Parrales

La obesidad es, al menos en la mayoría de los casos, el resultado de cambios en los estilos de vida de las personas y de los grupos sociales, Neisner, et al. (2003), Tackling Obesity in England, (2001).

El niño se encuentra inmerso en la sociedad de la superabundancia y de la disponibilidad total de alimento, en muchas ocasiones "comida basura". La obesidad tiene una etiología multifactorial, donde la libre disponibilidad de alimentos, los cambios en los hábitos alimentarios, el sedentarismo o los factores psicológicos y sociales tienen una importancia fundamental, Alustiza, (2004).

La persistencia de la obesidad infantil hasta la edad adulta aumenta significativamente el riesgo de padecer diabetes, enfermedad cardiovascular, hipertensión arterial, colecistitis y colelitiasis, Dietz, (1998).

Los estudios epidemiológicos sugieren que las causas principales están relacionadas con los cambios ambientales y de los estilos de vida ocurridos en las últimas décadas, Gutiérrez, (2005).

En 2004 el Ministerio de Sanidad y Consumo ha elaborado la Estrategia para la Nutrición, Actividad Física y Prevención de la Obesidad (NAOS) cuya misión es servir de plataforma donde incluir e impulsar todas aquellas iniciativas que contribuyan a lograr el necesario cambio social en la promoción de una alimentación saludable y la prevención del sedentarismo. Dentro de esta estrategia se nos pide a los pediatras de AP un papel protagonista en la prevención de la obesidad infantil, reconocer la obesidad como enfermedad crónica y 


\section{Enfoque sobre los factores de riesgo más relevantes de la diabetes en niños}

Vol. 2, núm. 4., (2018)

Colombo Efraín Arteaga Delgado; Raúl Ivan Casahualpa Leones; Fabrico Wladimir Gutiérrez Rivera; Jenniffer Betzabe Santistevan Holguin; Yuly Simone Mitte Baque; Alexandra Anabell

Figueroa Parrales

detectarla sistemáticamente en nuestras consultas, sobre todo en niños potencialmente en riesgo: sobrepeso o bajo peso al nacimiento, hijos de madres diabéticas o fumadoras durante el embarazo, obesidad en los padres, Fernández, (2005).

El sobrepeso y la obesidad son condiciones desfavorables para la salud, resultado del balance positivo de energía que se traduce en un acúmulo de grasa corporal. El estilo de vida actual se caracteriza por ingerir mayor cantidad de alimentos (energía), realizar cada vez menos ejercicio y pasar muchas horas laborales y recreativas sin llevar a cabo algún tipo de actividad física (sedentarismo), lo cual contribuye al sobrepeso y la obesidad. Estas condiciones predisponen a los individuos a padecer numerosas enfermedades crónicas, como la diabetes tipo 2 (DT2) y enfermedades cardiovasculares, responsables de las principales complicaciones y causas de mortalidad en la edad adulta, Peralta, et al. (2014).

La obesidad es una enfermedad crónica que de por vida requiere actividad física sistemática y hábitos de alimentación adecuados. Para que los programas de control de peso en el escolar den buenos resultados, éste y su familia deben seguir trabajando activamente para modificar los hábitos y se pueda mantener el peso ideal, mediante un plan de alimentación personalizada y un programa de ejercicio físico que disminuyan el índice de masa corporal y, con esto, los riesgos de enfermedades cardiovasculares, dislipidemia y diabetes mellitus, Barlow, Dietz, (1998) y Arellano, et al. (2004).

La obesidad general y central se genera por el aumento de triglicéridos, glucosa y presión arterial, así como la disminución del colesterol HDL, condiciones que engloban el llamado 


\section{Enfoque sobre los factores de riesgo más relevantes de la diabetes en niños}

Vol. 2, núm. 4., (2018)

Colombo Efraín Arteaga Delgado; Raúl Ivan Casahualpa Leones; Fabrico Wladimir Gutiérrez Rivera; Jenniffer Betzabe Santistevan Holguin; Yuly Simone Mitte Baque; Alexandra Anabell Figueroa Parrales

síndrome metabólico. Este síndrome cursa con hiperinsulinemia e intolerancia a la glucosa presentes desde la niñez, la adolescencia o en la vida adulta, Peralta, et al. (2014).

En niños hispanos con sobrepeso e historia familiar de DT2, se han encontrado alteraciones en la tolerancia a la glucosa en $28 \%$ de la población, independientemente del grado de obesidad. De estos, el $41 \%$ estuvieron expuestos a diabetes gestacional, asociados con resistencia a la insulina y con una función deficiente de las células $\beta$ del páncreas. La hipertensión arterial es cada vez más frecuente en la población pediátrica y está asociada con obesidad e historia familiar de hipertensión, Peralta, et al. (2014).

La obesidad constituye el elemento que con mayor frecuencia se asocia con incremento en la incidencia de diabetes en los jóvenes. Está demostrado que la pérdida de peso mejora la sensibilidad a la insulina en niños y adolescentes. (Rochinni demostró que la pérdida de peso mejoró la sensibilidad a la insulina en ayuno en un grupo de 50 adolescentes obesos). Aunque hasta donde se sabe no existe una gran diferencia entre una dieta sana y una que no lo es, parece que los jóvenes con diabetes albergan un concepto negativo sobre la primera. Está demostrado que la epidemia de obesidad tiene una correlación lineal con la ingestión de bebidas azucaradas, las horas que se pasan frente a una pantalla y la disminución de la actividad física. La tarea del educador en diabetes deberá ser la de un facilitador efectivo para modificar dicha percepción, Frenk, Márquez, (2010).

La disputa sobre los factores ambientales asociados a la inmunopatogenia de la DM1 es un tema altamente controversial, en el cual aún no hay un consenso pleno a la vista. Es por ello que el análisis de la interacción ambiental y genética en la DM1 no debe manejarse como una 


\section{Enfoque sobre los factores de riesgo más relevantes de la diabetes en niños}

Vol. 2, núm. 4., (2018)

Colombo Efraín Arteaga Delgado; Raúl Ivan Casahualpa Leones; Fabrico Wladimir Gutiérrez Rivera; Jenniffer Betzabe Santistevan Holguin; Yuly Simone Mitte Baque; Alexandra Anabell

Figueroa Parrales

asociación estadística, hay que evaluar en qué punto de la fisiología del sistema inmune encajan y son capaces de explicar las características observadas en dichos pacientes. Es fácil caer en el error de defender un punto de la historia natural de la enfermedad, pero la tendencia actual es tratar de llegar a una teoría unificadora la cual permita incluir aspectos influyentes pero que radique en una línea principal de ideación patológica, Aguirre, et al. (2012).

El aumento del nivel económico ha modificado la accesibilidad a los alimentos. El desarrollo tecnológico ha hecho innecesario el desarrollo de esfuerzo físico en la vida diaria de la mayoría de las personas. Ahora nos vemos ante la tesitura de intentar dar marcha atrás en unas urbes hipertrofiadas, llenas de vehículos, sin espacio para el caminante. En un mercado de la alimentación en el que predominan las ofertas y la publicidad sobre productos que proporcionan aportes energéticos muy superiores a lo necesario, pero que el público ha ido asimilando como normal, natural o legítimo consumir. En un contexto social en el que las familias han modificado su estructura y relaciones de forma sustancial, Martínez, (2005).

Dado que el estilo de vida es un conjunto de decisiones que puede afectar o beneficiar a la salud con algún grado de control voluntario, se espera que constituya un factor a favor del control del paciente portador de DM, sobre todo en los casos en que es parte de una estrategia educativa de la atención médica. Investigaciones argumentan la relación existente entre los malos estilos de vida y el mal control glucémico, así, la alimentación, el control del estrés y el sedentarismo son factores que guardan una importante relación - como parte del estilo de vidacon esta enfermedad, los cuales pueden ser modificados, para luchar en contra las tendencias actuales, tanto de entretenimiento como de alimentación. Todos estos, factores que deberán ser 


\section{Enfoque sobre los factores de riesgo más relevantes de la diabetes en niños}

Vol. 2, núm. 4., (2018)

Colombo Efraín Arteaga Delgado; Raúl Ivan Casahualpa Leones; Fabrico Wladimir Gutiérrez Rivera; Jenniffer Betzabe Santistevan Holguin; Yuly Simone Mitte Baque; Alexandra Anabell Figueroa Parrales

tomados en cuenta para otras investigaciones, así como el control de variables que no permitan demostrar una relación proporcional entre las empleadas en este estudio, Urbán, et al. (2015).

Cuando la causa que motiva la ansiedad no se identifica, la persona entra en un estado de ansiedad continua, que de forma muy frecuente alivia comiendo. Entre los factores de riesgo para obesidad destacan los antecedentes familiares, bases culturales, sedentarismo y ansiedad, entre otros. Los obesos suelen presentar, además, características psicológicas que tienden a agravar su problema, Silvestre y Stavile, (2005).

Entre los estilos de vida analizados, se observó que a partir de los 6 años la prevalencia de obesidad era más elevada en los niños y jóvenes que aportaban mayor proporción de energía a partir de la ingesta grasa (más de $38 \%$ kcal) en relación con los que realizaban ingestas porcentuales de grasa más bajas. Este hecho se observó también en el subgrupo femenino entre 14 y 17 años, Aranceta, et al. (2005).

En los varones a partir de los 14 años se apreció una diferencia estadísticamente significativa en el consumo de productos azucarados, bollería, embutidos y refrescos azucarados entre los obesos y los no obesos. Las tasas de prevalencia de obesidad eran más elevadas en los chicos con edades entre 6 y 14 años que realizaban con mayor frecuencia consumos de embutidos. Por la prevalencia de obesidad fue inferior en los niños y jóvenes ubicados en el cuartil más alto de la distribución de consumo de frutas y verduras (4 ó más raciones al día). Los chicos y chicas que realizan un desayuno completo expresan tasas de obesidad más bajas que los que no desayunan o realizan un desayuno incompleto, Aranceta, et al. (2005). 


\section{Enfoque sobre los factores de riesgo más relevantes de la diabetes en niños}

Vol. 2, núm. 4., (2018)

Colombo Efraín Arteaga Delgado; Raúl Ivan Casahualpa Leones; Fabrico Wladimir Gutiérrez Rivera; Jenniffer Betzabe Santistevan Holguin; Yuly Simone Mitte Baque; Alexandra Anabell

Figueroa Parrales

Considerando globalmente el tiempo medio diario dedicado al desempeño de actividades sedentarias (estudio, televisión, ordenador, videojuegos...), la prevalencia de obesidad fue más elevada entre los chicos y chicas que dedican mayor tiempo a este tipo de actividades en comparación con los que dedican menos tiempo. La prevalencia de obesidad era más elevada en las chicas que dedicaban más de 2 horas diarias a ver la televisión en comparación con las que dedicaban menos de 1 hora a esta actividad. La prevalencia de obesidad entre los chicos y chicas que no practicaban ningún deporte habitualmente fue más elevada en comparación con los que sí tenían este hábito, especialmente entre los chicos que practicaban actividades deportivas 3 veces a la semana, Aranceta, et al. (2005).

La diabetes tipo 2, el cáncer y todas las enfermedades cardiovasculares son de las patologías crónicas que más recursos consumen. Y algunos de sus factores determinantes son prevenibles. Por ejemplo, la obesidad, el sedentarismo y el consumo de tabaco. Los estilos de vida parecen estar en la base de muchos de estos problemas y pueden ser modificados, Martínez, (2005).

Las dietas con alto contenido de grasas saturadas, carbohidratos de rápida absorción y poco consumo de grasas polinsaturadas y fibras, así como el incremento de sedentarismo, son algunas características de la transición epidemiológica nutricional, asimismo la mayor disponibilidad de alimentos de bajos costo ha permitido que la población pueda acceder a alimentos con alto contenido energético, Orden, et al. (2005).

Un estudio sobre la actividad física y hábitos alimentarios en niños obesos pertenecientes a un nivel socioeconómico bajo, reporto un bajo consumo de lácteos, verduras y frutas y un 


\section{Enfoque sobre los factores de riesgo más relevantes de la diabetes en niños}

Vol. 2, núm. 4., (2018)

Colombo Efraín Arteaga Delgado; Raúl Ivan Casahualpa Leones; Fabrico Wladimir Gutiérrez Rivera; Jenniffer Betzabe Santistevan Holguin; Yuly Simone Mitte Baque; Alexandra Anabell Figueroa Parrales

elevado consumo de bebidas azucaradas y alimentos con alta densidad energética. Más del 55\% de los niños no realizaban actividades físicas fuera del colegio. Los resultados indicaron deficiencias en las motivaciones y barreras para adquirir hábitos saludables de alimentación y de actividad física. A su vez, las madres mostraron poca motivación y baja predisposición para apoyar a sus hijos, Olivares, et al. (2006).

Los datos de vigilancia epidemiológica que disponemos, a través de las estadísticas del Ministerio de Salud, demuestran que la población infantil, de menores de 6 años, presenta cifras cada vez menores de bajo peso y un incremento importante en los niveles de malnutrición por exceso. Esta realidad no es ajena a lo que sucede en otros países en desarrollo o desarrollados, en los que al igual que en nuestro país, los cambios desfavorables en los estilos de vida están determinando un perfil epidemiológico caracterizado por el sedentarismo, los malos hábitos alimentarios y las enfermedades crónicas no transmisibles (ECNTs). Estas patologías, que representan una importante carga en morbilidad y mortalidad en la edad adulta, se originan en gran parte, muy precozmente en la etapa infantil, incluso antes, en la etapa fetal, y se incrementan durante toda la vida, Rodríguez, (2007).

La actividad física de los padres, como ejemplo a seguir por sus hijos y de los propios niños, estimulando una vida activa con actividad física adecuada a cada una de las etapas de su maduración y limitando las actividades sedentarias, Chiesa et al. (2009).

El riesgo a causa del sedentarismo, el estilo de vida sedentario, o mejor dicho, el inactivo. Este estilo de vida es una característica común del estilo de vida de sociedades desarrolladas y es aún más pronunciado en los países mediterráneos del sur de Europa. Respecto a la población 


\section{Enfoque sobre los factores de riesgo más relevantes de la diabetes en niños}

Vol. 2, núm. 4., (2018)

Colombo Efraín Arteaga Delgado; Raúl Ivan Casahualpa Leones; Fabrico Wladimir Gutiérrez Rivera; Jenniffer Betzabe Santistevan Holguin; Yuly Simone Mitte Baque; Alexandra Anabell

Figueroa Parrales

pediátrica, situaciones como la del menor nivel de seguridad vial y cívica ha hecho que el porcentaje de niños que viajan a la escuela a pie o en bicicleta o jugando en las calles, parques o los espacios públicos son más bajos. Este hecho podría ser incluido en el llamado "efecto canguro protector" que muchos padres adoptan impidiendo que sus hijos jueguen y corren por las calles "por su cuenta". Varela et al. (2013).

Junto al Los avances en las nuevas tecnologías hacen que las personas se involucren. Más y más en actividades de muy bajo consumo de energía. Durante su tiempo libre (consola de juegos, video juegos, internet, etc.). Debemos recordar que el comportamiento sedentario no es solo menor actividad física, pero un conjunto de actitudes individuales es que el hecho de estar sentado y/o tendido Abajo se convierte en el comportamiento postural predominante lo que implica un gasto energético muy limitado, Varela et al. (2013).

Los comportamientos sedentarios están presentes en muchos lugares y situaciones: el lugar de trabajo, el entorno escolar, Transporte, o tiempo libre y de ocio. El problema es que una serie de estudios realizados en los últimos años han demostrado que el sedentarismo o la inactividad física son un factor de riesgo para el desarrollo de muchas enfermedades crónicas, Varela et al. (2013).

Según Chiesa et al. (2009), el sedentarismo es causado por la vida moderna (computadores personales, video juegos, acceso a Internet, videocasete, DVD, etcétera) ha promovido la disminución de los tiempos de recreación física de los niños (juegos en la calle, plazas, etcétera). La prevención de esta situación requiere: 


\section{Enfoque sobre los factores de riesgo más relevantes de la diabetes en niños}

Vol. 2, núm. 4., (2018)

Colombo Efraín Arteaga Delgado; Raúl Ivan Casahualpa Leones; Fabrico Wladimir Gutiérrez Rivera; Jenniffer Betzabe Santistevan Holguin; Yuly Simone Mitte Baque; Alexandra Anabell Figueroa Parrales

- Realizar actividad física 60 minutos, diarios o en días alternos;

- Limitar actividades sedentarias a menos de dos horas por día;

- Fomentar la participación en ejercicios recreativos y/o competitivos adecuados a cada paciente en particular.

La actividad física es muy difícil de cuantificar, pero es notorio que en los niños y adolescentes ha disminuido en las últimas décadas por múltiples motivos. El rápido desarrollo de la tecnología ha determinado que los individuos prácticamente no realicen ningún esfuerzo en la vida diaria, la presencia de televisión, computadoras y videojuegos han sustituido los juegos al aire libre y llevan a los niños a recrearse en forma sedentaria. Los juegos, la actividad física y el deporte constituyen una fuente de salud y desarrollo para todos los individuos, Gambetta, et al. (2005).

En el riesgo de la enfermedad cardiovascular la prevención cardiovascular se debe iniciar precozmente, tanto en niños con enfermedades que determinan situaciones especiales de alto riesgo como en aquellos que por su exposición a los factores de riesgo cardiovascular tienen un mayor riesgo de padecer enfermedad cardiovascular en su vida adulta. Los factores de riesgo comienzan a actuar desde edades tempranas, ya sea por exposición a hábitos no saludables de vida (mala alimentación, sedentarismo, tabaquismo) y/o a los clásicos factores de riesgo cardiovascular. La prevención se debe realizar en todas las edades, Chiesa et al. (2009).

La práctica regular de ejercicio físico es una recomendación establecida para prevenir y tratar los principales factores de riesgo cardiovascular modificables, como la diabetes mellitus, la hipertensión y la dislipemia. Realizar actividad física de intensidad moderada durante un mínimo 


\section{Enfoque sobre los factores de riesgo más relevantes de la diabetes en niños}

Vol. 2, núm. 4., (2018)

Colombo Efraín Arteaga Delgado; Raúl Ivan Casahualpa Leones; Fabrico Wladimir Gutiérrez Rivera; Jenniffer Betzabe Santistevan Holguin; Yuly Simone Mitte Baque; Alexandra Anabell

Figueroa Parrales

de 30 min 5 días por semana o de intensidad alta durante un mínimo de 20 min 3 días por semana mejora la capacidad funcional y se asocia a reducciones en la incidencia de enfermedad cardiovascular y mortalidad. Cordero et al, (2014).

Se ha mostrado que llevar una vida físicamente activa implica muchos Beneficios para la salud: disminuye el riesgo de mortalidad enfermedades cardiovascular, previene y / o retrasa la desarrollo de la hipertensión arterial, mejora la perfil lipídico sanguíneo (reduce triglicéridos y aumenta el colesterol HDL), disminuye el riesgo de padecen diabetes tipo 2, e incluso ciertos tipos De cánceres (colon, cáncer de mama), mejora el cuerpo. Control de peso (prevención y tratamiento del sobrepeso y obesidad), ayuda a optimizar y mantener el fuerza y resistencia muscular, etc., Varela et al. (2013).

No se dispone de evidencia científica sobre la relación entre los estilos de vida y la aparición de DM1 pero la prevención primaria es eficaz para prevenir la aparición de la DM2, Australian Centre for Diabetes Strategies, (2001).

El diagnóstico de la DM en los niños y en los adolescentes se basa generalmente en los síntomas y signos característicos de la enfermedad: poliuria, polidipsia y pérdida de peso; así como en la determinación al azar de la glucosa en el plasma venoso de concentraciones superiores a $200 \mathrm{mg} / \mathrm{dL}(11,1 \mathrm{mmol} / \mathrm{L})$, sin cetonuria. Otra posibilidad es la presencia de cifras de glicemia en ayunas mayores que $140 \mathrm{mg} / \mathrm{dL}$ en dos ocasiones y dos sobrecargas por la vía oral de glucosa con una glicemia a las 2 horas en un punto mayor a $200 \mathrm{mg} / \mathrm{dL}$ (utilizar 1,75 g/kg, hasta un máximo de 75 g de glucosa). En ocasiones se presenta con una descompensación aguda, Batista, et al. (1998). 


\section{Enfoque sobre los factores de riesgo más relevantes de la diabetes en niños}

Vol. 2, núm. 4., (2018)

Colombo Efraín Arteaga Delgado; Raúl Ivan Casahualpa Leones; Fabrico Wladimir Gutiérrez Rivera; Jenniffer Betzabe Santistevan Holguin; Yuly Simone Mitte Baque; Alexandra Anabell Figueroa Parrales

Dislipemias, se definen como alteraciones en la concentración de lípidos en sangre. La prevalencia en niños es desconocida. Algunos estudios han encontrado una prevalencia de entre 5 a $12 \%$. Se recomiendan estrategias a nivel poblacional con el objetivo de mantener los niveles normales de lípidos en sangre en todos los niños, y estrategia individual con el objetivo de identificar y tratar niños y adolescentes en riesgo de tener niveles de colesterol elevados, Gambetta, et al. (2005).

Gambetta, et al. (2005), El estudio de perfil lipídico en la edad pediátrica sólo debe realizarse en niños mayores de dos años y adolescentes en los que existan:

a) historia familiar positiva: padres con colesterol total mayor o igual a $240 \mathrm{mg} / \mathrm{dl}$; antecedentes familiares (padres o abuelos) de enfermedad cardíaca prematura (<55años) que incluye patología coronaria, muerte súbita cardíaca; enfermedad vascular periférica y enfermedad cerebro vascular.

b) niños con otros factores de riesgo: hipertensión, obesidad, tabaquismo, sedentarismo, alcoholismo, medicación que se asocia con hiperlipemia (ácido retinoico, anticonceptivos orales, anticonvulsivantes), diabetes mellitus y síndrome nefrótico; y en los que se desconocen los antecedentes familiares.

\section{Conclusiones.}

En los niños la Diabetes Mellitus del tipo 1 se presenta por distintos factores de riesgo, caracterizado por el sedentarismo en la actualidad una de las principales fuentes de amenaza para la salud de los niños, los malos hábitos alimentarios nutricionales e inadecuados, la obesidad en 


\section{Enfoque sobre los factores de riesgo más relevantes de la diabetes en niños}

Vol. 2, núm. 4., (2018)

Colombo Efraín Arteaga Delgado; Raúl Ivan Casahualpa Leones; Fabrico Wladimir Gutiérrez Rivera; Jenniffer Betzabe Santistevan Holguin; Yuly Simone Mitte Baque; Alexandra Anabell

Figueroa Parrales

la población pediátrica por los antecedentes familiares de obesidad, el sobrepeso, los problemas cardiovasculares, la hipertensión. La diabetes en niños se ha identificado como un problema global que se ha complicado por varios factores ambientales y genéticos, las enfermedades crónicas no transmisibles (ECNTs). Estas patologías, que representan una importante carga en morbilidad y mortalidad en la edad adulta, se originan en gran parte, muy precozmente en la etapa infantil, incluso antes, en la etapa fetal, y se incrementan durante toda la vida.

\section{Referencias Bibliográficas}

ADA, (2008), Standards of medical care in diabetes. Diabetes Care. 2008; American Diabetes Association, 31 (Suppl 1): 512-54.

ADA. (2000), Type 2 Diabetes in Children and Adolescents, Diabetes Care, La American Diabetes Association (ADA) 23(3): 381-389.

Aguirre, M., Rojas, J., Cano, R., Villalobos, M., Paoli, M. y Berrueta, L., (2012), Diabetes mellitus tipo 1 y factores ambientales: la gran emboscada, Revista Venezolana de Endocrinología y Metabolismo - Volumen 10, Número 3: 122-135.

Almaguer, A., Miguel, P., Será, C., Mariño, A., Oliveros, R., (2012), Actualización sobre diabetes mellitus, Universidad de Ciencias Médicas de Holguín, Correo Científico Médico, 16 (2), ISSN 1560-4381.

Alustiza, E., (2004), Prevención y tratamiento de la obesidad en Atención Primaria. AVPAP [En línea] Disponible en www.avpap.org/documentos/donostia2004/alustiza.htm

Aranceta, J., Pérez, C., Ribas, L., Serra, L., (2005), Epidemiología y factores determinantes de la obesidad infantil y juvenil en España, Revista Pediatría de Atención Primaria Vol. VII, Suplemento 1: S13-20.

Arellano, M., Bastarrachea, S., Bourges, R., (2004), Grupo para el Estudio y Tratamiento de la Obesidad en Adolescentes, La obesidad en México, Rev Endocrinol Nutr. 12 (4) Supl 3: S80-S87.

Australian Centre for Diabetes Strategies, (2001), National Evidence Based Guidelines for the management of type 2 diabetes mellitus primary prevention, Case detection and diagnosis. National Health and Medical Research Council (NHMRC). 


\section{Enfoque sobre los factores de riesgo más relevantes de la diabetes en niños}

Vol. 2, núm. 4., (2018)

Colombo Efraín Arteaga Delgado; Raúl Ivan Casahualpa Leones; Fabrico Wladimir Gutiérrez Rivera; Jenniffer Betzabe Santistevan Holguin; Yuly Simone Mitte Baque; Alexandra Anabell Figueroa Parrales

Batista, R., Ortega, L., Fernández, G., (1998), Diabetes mellitus. Manejo y consideraciones terapéuticas, RESUMED 11(1): 6-23

Barja, S., Arteaga, A., Acosta, A. y Col. (2004), Resistencia Insulìnica y otras expresiones del Sìndrome Metabólico en niños y adolescentes chilenos. Rev. Med. Chile. www.labnutricion.cl/resistencia_chicosobesos.htm.

Barlow, S., Dietz, W., (1998), Expert Committee Recommendations, Obesity evaluation and treatment, Pediatrics 102:1-11.

Blommgarden, Z., (2004), Type 2 diabetes in the young: the evolving epidemic, Diabetes Care, 27: $998-1010$.

Botero, D., Wolfsdorf, I., (2005), Diabetes Mellitus in Children and Adolescents, Review Article, Arch Med Res, 36: 281-290.

Calzada, R., (2003), Prevalencia y etiopatogenia, en: Calzada, R., editor. Obesidad en niños y adolescentes, Editores de Textos Mexicanos, México, p.15-19.

Cordero, A., Masiá, M., Galve, E., (2014), Ejercicio físico y salud, Revista Española de Cardiología (English Edition), Volume 67, Issue 9, Pages 748-753, https://doi.org/10.1016/j.recesp.2014.04.007Get rights and content

Chiesa, P., Gambetta, J., Dutra, S., (2009), Prevención cardiovascular desde la edad pediátrica, Revista uruguaya de cardiología, Volumen $24 \mathrm{~N}^{\circ} 2,105-111$

Chukwueke, I., Cordero, Z., (2010), Overview of type 2 diabetes in Hispanic Americans, Int J Body Compos Res, 8 (Supp): 77-81.

Chu, N., (2001), Prevalence and trends of obesity among school children in Taiwan $Đ$ the Taipei Children Heart Study, Int J Obes, 25: 170-176.

Del Águila, C., (2017), Obesidad en el niño: factores de riesgo y estrategias para su prevención en Perú, Rev Perú Med Exp Salud Pública, 34(1): 113-8, doi: 10.17843/rpmesp.2017.341.2773

Dietz, W., (1998), Childhood weight affects adult morbidity and mortality, J Nutr. 128: 411-414.

Fernández, M., (2005), Experiencias de tratamiento integral de la obesidad infantil en pediatría de Atención Primaria, Revista Pediatría de Atención Primaria, Vol. VII, Suplemento 1: S35-47.

Frenk, P., Márquez, E., (2010), Diabetes mellitus tipo 2 en niños y adolescentes. Medicina Interna de México Volumen 26, núm. 1: 36-47.

Revista Científica Mundo de la Investigación y el Conocimiento. 2 (4). pp. 270-297 


\section{Enfoque sobre los factores de riesgo más relevantes de la diabetes en niños}

Vol. 2, núm. 4., (2018)

Colombo Efraín Arteaga Delgado; Raúl Ivan Casahualpa Leones; Fabrico Wladimir Gutiérrez Rivera; Jenniffer Betzabe Santistevan Holguin; Yuly Simone Mitte Baque; Alexandra Anabell

Figueroa Parrales

Gagliardino, J., (1997), Cómo tratar mi Diabetes dedicado a personas con diabetes no insulinodependientes, Ed. Boehringer Mannheim GMBH, La Plata Argentina.

Gambetta, J., Satriano, R., Tabarez, A. y otros, (2005), Promoción de la salud cardiovascular en la infancia, Arch Pediatr Urug 2005; 76(1): 51-58

Godínez, S., Mormolejo, O., Márquez, R., Siordia, V., Baeza, C., (2002), La grasa visceral y su importancia en obesidad, Rev Endocrinol Nutr, 10(3)121-127.

Gutiérrez, M., (2005), Prevención de la obesidad y promoción de hábitos saludables, Foro Pediátrico, 2 (Supl 1): 54-59.

Hernández, B., Cuevas, L., Shamah, T., Monterrubio, E., Ramírez, C., García, R., Rivera, J., Sepúlveda, J., (2003), Factores asociados con sobrepeso y obesidad en niños mexicanos de edad escolar: resultados de la Encuesta Nacional de Nutrición 1999. Salud Pública México, 45(Supl 4): S551-S557.

Hornell, A., Lagstrom, H., Lande, B., Thorsdottir, I., (2013), Protein intake from 0 to 18 years of age and its relation to health: a systematic literature review for the Nordic Nutrition Recommendations. Food Nutr Res, 57: 210-23, doi:10.3402/fnr.v57i0.21083. Print 2013.

IDF. Atlas Diabetes (2011), disponible en Atlas Diabetes 2011 http://www.idf.org/diabetesatlas/

Ludwig, D., Ebbeling, C., (2001), Type 2 Diabetes mellitus in Children, JAMA, 286 (12): 1427 1430.

Luna, M., Rangel, D., Guizar, J., Amador, N., (2007), Modificación de factores de riesgo para desarrollar diabetes mellitus tipo 2 en escolares obesos, Revista Médica del Instituto Mexicano del Seguro Social, 45 (1): 53-62.

Maffeis, C., Pietrobelli, A., Grezzani, A. y Col. (2001), Waist Circunference and Cardiovascular Risk Factors in Prepuberal Children. Obesity Research; 9: 179-187.

Manco, M., Dallapiccola, B., (2012), Genetics of pediatric obesity, Pediatrics, 130(1): 123-33, doi: 10.1542/peds.2011-2717.

Martínez, J., Villarino, A., García, R., Calle, M., Marrodán, M., (2013), Obesidad infantil en España: hasta qué punto es un problema de salud pública o sobre la fiabilidad de las encuestas, Nutrición clínica y dietética hospitalaria, 33(2): 80-88 DOI: $10.12873 / 332$ obesidadspain

Martínez, A., (2005), Prevención integral de la obesidad infantil: el Plan Andaluz, Revista Pediatría de Atención Primaria, Vol. VII, Suplemento 1, S21-34. 


\section{Enfoque sobre los factores de riesgo más relevantes de la diabetes en niños}

Vol. 2, núm. 4., (2018)

Colombo Efraín Arteaga Delgado; Raúl Ivan Casahualpa Leones; Fabrico Wladimir Gutiérrez

Rivera; Jenniffer Betzabe Santistevan Holguin; Yuly Simone Mitte Baque; Alexandra Anabell

Figueroa Parrales

McKnight, H., Sababu, S., Kelly, S., (2005), The care of Children and Adolescents with Type 2 Diabetes, Journal of Pediatric Nursing, 20: 96-106.

Michaelsen, K., Greer, F., (2014), Protein need early in life and long-term health, Am J Clin Nutr 99(3): 718S-22S, doi: 10.3945/ajen.113.072603

Morrison, J., (2000), Utility of difeferent measures of body fat distribution in children and adolescents, Am. J. Epidemiol, 152: 1179-1184

Neisner, J., Histon, T., Goeldner, J., Moon, C., (2003), Background paper on the prevention and treatment of overweight and obesity, Kaiser Permanente's Care Management Institute, Disponible en www.kpcmi.org

Olivares, S., Bustos, N., Moreno, X., Lera, L., Cortez, S., (2006), Actitudes y prácticas sobre alimentación y actividad física en niños obesos y sus madres en Santiago, Rev chil Nutr. Chile. 33(2): 170-9.

OPS, (2008), Guías ALAD de diagnóstico, control y tratamiento de la Diabetes Mellitus Tipo 2, Washington, D.C.: Organización Panamericana de la Salud (OPS), Disponible en: http://www.paho.org/Spanish/AD/DPC/NC/diaguia-alad.pdf

Orden, A., Torres, M., Luis, M., Cesani, M., Quintero, F., Oyhenart, E., (2005), Evaluación del estado nutricional en escolares de bajos recursos socioeconómicos en el contexto de la transición nutricional, Arch Argent Pediatr. 103(3): 205-11.

Padrón, R., (2008), Diabetes Mellitus, Propedéutica clínica y fisiopatología odontológica fundamental. Editorial Ciencias Médicas, La Habana, 215-21.

Peralta, J., Gómez, J., Estrada, B., Karam, R., Cruz, M., (2014), Genética de la obesidad infantil, Revista Médica del Instituto Mexicano del Seguro Social, 52 Disponible en:<http://www.redalyc.org/articulo.oa?id=457745485014>ISSN 0443-5117.

Rodríguez, L., (2007), Situación nutricional del escolar y adolescente en Chile, Educación Continua, Revista Chilena de Pediatría, 78 (5): 523-533

Rolland, C., Deheeger, M., Maill, T., Bellisle, F., (2006), Early adiposity rebound: causes and consequences for obesity in children and adults, Int J Obes (Lond), 30 (Suppl 4): S11-S17. doi:10.1038/sj.ijo.0803514.

Serra, L., Ribas, L., Aranceta, J., Pérez, C., Saavedra, P., Peña, L., (2003), Obesidad infantil y juvenil en España, Resultados del Estudio enKid (1998-2000), Medicina Clínica Barcelona, 121: 725-732.

Revista Científica Mundo de la Investigación y el Conocimiento. 2 (4). pp. 270-297 


\section{Enfoque sobre los factores de riesgo más relevantes de la diabetes en niños}

Vol. 2, núm. 4., (2018)

Colombo Efraín Arteaga Delgado; Raúl Ivan Casahualpa Leones; Fabrico Wladimir Gutiérrez Rivera; Jenniffer Betzabe Santistevan Holguin; Yuly Simone Mitte Baque; Alexandra Anabell

Figueroa Parrales

Silvestre, E., Stavile, A., (2005), Aspectos psicológicos de la obesidad, Importancia de su identificación y abordaje dentro de un enfoque interdisciplinario, Posgrado en obesidad a distancia, Universidad Favaloro, Córdoba.

Steinberger, J., Stephen, R., (2003), Obesity, Insulin Resistance, Diabetes, and Cardiovascular Risk in Children, DHA Scientific Statement An American Heart Association Scientific Statement From the Atherosclerosis, Hypertension, and Obesity in the Young Committee and the Diabetes Committee. Circulation 2003; 107: 1448

Tackling Obesity in England, (2001), Report by the Controller and Auditor General, HC 220 Session 2000-2001: London: The Stationery Office, Disponible en www.nao.org.uk/

Taksali, S., Yeckel, C., Allen, K., Lopes, M., Savoye, M., Morrison, J., Sherwin, R., Caprio, S., (2004), Obesity and the metabolic syndrome in children and adolescents, N Engl J Med. 350: 2362-2374.

Urbán, B., Coghlan, J., Castañeda, O., (2015), Estilo de vida y control glucémico en pacientes con Diabetes Mellitus en el primer nivel de atención, Atención Familiar, 22(3): 68-71.

Varela, G., Alguacil, L., Alonso, E., Aranceta, J., Ávila, J., Aznar, S., Belmonte, S., et al. (2013), Obesidad y sedentarismo en el siglo XXI: ¿Qué se puede hacer y qué se debe hacer?, Nutr Hosp 2013; 28 (Supl. 5): 1-12 ISSN, (Versión electrónica): 1699-5198

WHO, (2002), The World Health Report: 2002: Reducing risks, promoting healthy life, World Health Organisation, Geneva.

WHO, (1998), Obesity, Preventing and managing the global epidemic, Report of a WHO consultation of obesity, World Heath Organization, Geneva.

Williams, C., Hayman, L., Daniels, S. y Col. (2002), Cardiovascular Health in Childhood. Statemen for Health Professionals From the Committee an Atheosclerosis, Hypertension, and Obesity in the Young of the Council on Cardiovascular Disease in the Young American Heart Association. Circulation 2002: 106:143 